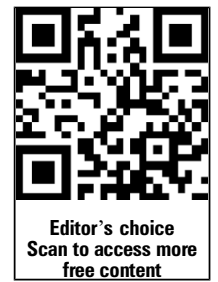

${ }^{1}$ Bradford Neonatology, Bradford Royal Infirmary, Bradford, West Yorkshire, UK ${ }^{2}$ Sheffield Children's Hospital, Western Bank, Sheffield, UK ${ }^{3}$ Department of Paediatrics, Pinderfields Hospital, Wakefield, UK

${ }^{4}$ Bradford Royal Infirmary, Bradford, UK

${ }^{5}$ School of Health Studies, University of Bradford, Bradford, West Yorkshire, UK Accepted 18 February 2013

\section{Correspondence to}

Dr Sam Joseph Oddie, Bradford Neonatology, Bradford Royal Infirmary, Duckworth Lane, BD16 1AR, Bradford, West Yorkshire BD9 6RJ, UK Sam.oddie@bthft.nhs.uk

Received 11 September 2012 Revised 7 February 2013 Accepted 18 February 2013 Published Online First 20 March 2013

\section{SLinked}

http://dx.doi.org/10.1136/ archdischild-2013-303898

To cite: Oddie SJ, Craven V, Deakin K, et al. Arch Dis Child Fetal Neonatal Ed 2013;98:F384-F387.

\title{
Severe neonatal hypernatraemia: a population based study
}

\author{
Sam Joseph Oddie, ${ }^{1}$ Vanessa Craven, ${ }^{2}$ Kathryn Deakin, ${ }^{3}$ Janette Westman, ${ }^{4}$ \\ Andrew Scally ${ }^{5}$
}

\section{ABSTRACT}

Aims To describe incidence, presentation, treatment and short term outcomes of severe neonatal hypernatraemia (SNH, sodium $\geq 160 \mathrm{mmol} / \mathrm{l}$ ). Methods Prospective, population based surveillance study over 13 months using the British Paediatric Surveillance Unit. Cases were $>33$ weeks gestation at birth, fed breast or formula milk and $<28$ days of age at presentation.

Results Of 62 cases of SNH reported $(7,95 \% \mathrm{Cl} 5.4$ to 9.0 per 100000 live births), 61 mothers had intended to achieve exclusive breast feeding. Infants presented at median day 6 (range 2-17) with median weight loss of 19.5\% (range 8.9-30.9). 12 had jaundice and 57 weight loss as a presenting feature. 58 presented with weight loss $\geq 15 \%$. 25 babies had not stooled in the $24 \mathrm{~h}$ prior to admission. Serum sodium fell by median $12.9 \mathrm{mmo} / / \mathrm{l}$ per $24 \mathrm{~h}$ (range $0-30$ ). No baby died, had seizures or coma or was treated with dialysis or a central line. At discharge, babies had regained $11 \%$ of initial birth weight after a median admission of 5 (range 2-14) days. 10 were exclusively breast fed on discharge from hospital.

Conclusions Neonatal hypernatraemia at this level, in this population, is strongly associated with weight loss. It occurs almost exclusively after attempts to initiate breast feeding, occurs uncommonly and does not appear to be associated with serious short term morbidities, beyond admission to hospital.

\section{INTRODUCTION}

Hypernatraemia occurring in newborns born at or near term has attracted significant clinical and research attention in recent years, largely as a result of cases of catastrophic weight loss associated with breastfeeding difficulty associated hypernatraemia $(\mathrm{BDAH})$, with or without severe consequences. ${ }^{1-8}$ In particular, a regional paediatric intensive care centre published a series of five seriously ill babies from a 4-year period emanating from a large geographical area. ${ }^{1}$ Published reports are mostly small case series, without clearly defined population based denominators. While some authors have expressed the view that the problem was of increasing prevalence, little or no hard evidence is published to support this view, with most reports not being based on a geographical population..$^{5} 910$ Although a standard definition of hypernatraemia (highest serum sodium $\geq 150 \mathrm{mmol} / \mathrm{l}$ ) has become common in such reports the inclusion of milder levels of dehydration hypernatraemia may be a problem. $^{2}{ }^{11}{ }^{12}$ Higher numbers of cases may be ascertained and reported where hypernatraemia is routinely sought by a blood test in babies with unusual weight loss. $^{9}{ }^{11} 12$ In part this

\section{What is already known on this topic}

Breastfeeding difficulty associated hypernatraemia (BDAH) occurs where weight loss, breastfeeding difficulty and hypernatraemia coexist in a newborn baby.

- BDAH is widely reported and is sometimes associated with morbidity.

\section{What this study adds}

- Severe hypernatraemia in newborn term babies is rare.

- Most infants with hypernatraemia present with weight loss and breastfeeding difficulty.

- Apart from the need for hospitalisation, short term clinical outcomes from BDAH appear to be good.

phenomenon explains the wide variety of prevalence figures for BDAH. ${ }^{2} 579$ 11-14

Furthermore, evidence suggests the extent of neonatal weight loss is substantially affected by hospital practices in support of effective breast feeding, which may in turn affect the prevalence of hypernatraemia. ${ }^{15} 16$

With these weaknesses in the published literature in mind, we chose to concentrate on the severe end of the hypernatraemia spectrum. We set out to obtain an estimate of the incidence of severe neonatal hypernatraemia $(\mathrm{SNH})$ in infants fed breast or formula milk in the UK and Republic of Ireland (ROI).

\section{METHODS}

We ascertained cases from 1 May 2009 to 1 June 2010 through the British Paediatric Surveillance Unit (BPSU). The BPSU facilitates active surveillance of rare disease via more than 3000 paediatricians in the UK and the ROI. Paediatricians are sent a monthly card and asked to say if they have seen any new cases of a 'menu' of rare conditions in the past month. Positive cases are followed up by the study team. Response rates to the monthly card are in excess of $90 \% .^{17}$

The reporting case definition used was 'a baby born at 34 or more weeks of gestation and seen in hospital with serum sodium $\geq 160 \mathrm{mmol} / \mathrm{l}$ at less than 29 days of age.'

Cases where the reporting clinician believed the hypernatraemia was a consequence of a urinary 
concentrating defect were excluded by the paediatricians or the study team.

Clinicians completed a case record form based on the clinical notes, encompassing feeding prior to presentation, clinical features and short term outcomes. Seizure diagnosis was based on clinical assessment.

Denominator data on live births were taken from the Office of National Statistics midyear estimates and Irish equivalent.

We conducted an internet survey of weighing practices in maternity units in mainland UK hospitals by sending a link to senior mid-wives and infant feeding coordinators in each maternity unit by email.

Data on the baby friendly accreditation status of the hospitals to which the cases were readmitted were abstracted from the website of the UK Baby Friendly Initiative (BFI). Comparison was made with the proportion of births which occur in fully accredited Baby Friendly Hospitals.

Ethical approval was obtained from the Bradford Research Ethics Committee, reference 08/H1302/129.

\section{ANALYSIS}

Data are presented as means and SDs or medians and ranges and IQRs for parametric and non-parametric data, respectively. The correlation between day of presentation and extent of weight loss and serum sodium was assessed using linear regression models using Stata (release 9.2, StataCorp, Texas, USA, 2006). Comparison of non-parametric variables was made using the Mann-Whitney U test. CIs for rates were by calculated using the Exact Binomial method.

\section{RESULTS}

In all, 62 confirmed cases of SNH resulted from 95 reports. A total of 18 were reported in error, five cases were duplicates, six paediatricians reported a case but were no longer contactable and four more paediatricians were unable to confirm the case on account of being unable to recall the patient's identifiers or obtain the notes. The response rate to the BPSU card during the study period was $93.8 \%$. Based on mid-term Office of National Statistics statistics, there were 880000 live births in the UK and ROI in the study period, leading to an incidence of SNH of 7 (95\% CI 5.4 to 9.0) per 100000 live births.

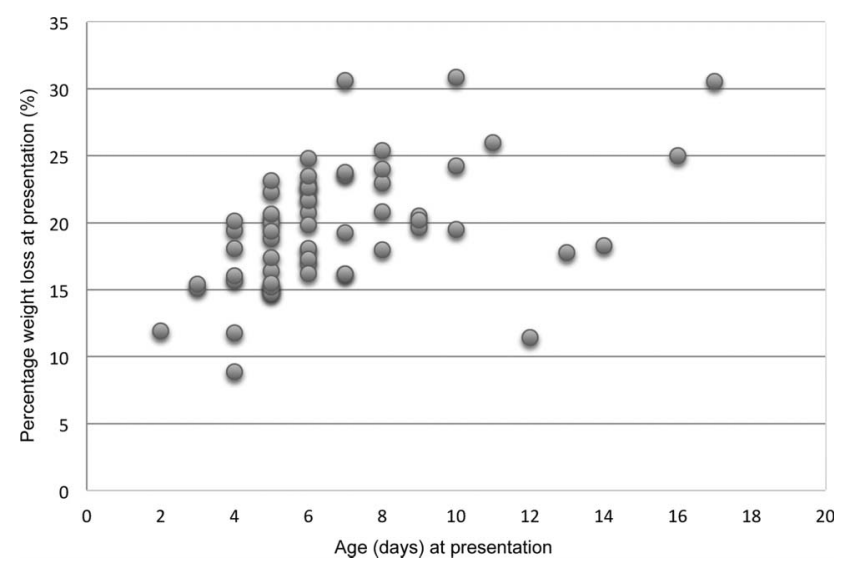

Figure 1 Weight loss against age at presentation in 62 cases of severe neonatal hypernatraemia in the UK and Republic of Ireland, May 2009 to June 2010 .

\section{Infant characteristics}

Overall, 26 women delivered by caesarean section, and nine by assisted vaginal delivery. A total of 52 had no previous live births. The median maternal age at delivery was 32 years (IQR 28-35). The median age of initial hospital discharge was 2 (IQR1-3) days (1 for normal vaginal deliveries).

The babies had normal birth weights (mean $3467 \mathrm{~g}$, SD $537 \mathrm{~g}$ ) with only one baby having birth weight $<2500 \mathrm{~g}$. No baby is known to have died during the admission or thereafter. Three babies were born between 35 and 37 completed weeks of gestation. One premature baby had Down syndrome and was readmitted with $23 \%$ weight loss at 6 days of age. One baby was noted to have a cleft palate and associated feeding problems at 18 days of age, after hypernatraemia was diagnosed.

\section{Presentation}

Clinician respondents described 61 babies as having been exclusively breast fed prior to admission. Only one case had been fed exclusively formula prior to developing hypernatraemia-this infant had signs of neonatal abstinence syndrome and had been observed for 5 days in hospital prior to discharge. A total of 55 $(89 \%)$ infants were reportedly only fed breast milk from the breast in the $24 \mathrm{~h}$ prior to diagnosis.

Eight babies presented before leaving hospital, of whom two did not meet the case definition on their first serum sodium measurement. In all, 54 babies were readmitted to hospital at a median 6 days of age (range 3-17, IQR 5-8). Six of the 54 readmitted babies developed more severe hypernatraemia, in one case from $181 \mathrm{mmol} / \mathrm{l}$ to $187 \mathrm{mmol} / \mathrm{l}$.

Median weight loss at presentation or readmission was $19.5 \%$ (range 8.9-30.9, IQR16.2-22.6) (see figure 1). Weight loss at presentation was significantly associated with age at presentation ( $\mathrm{r}=0.46, \mathrm{p}<0.0001)$.

The median highest serum sodium concentration was $164 \mathrm{mmol} / \mathrm{l}$ (range 160-187, IQR 161-168). In a multivariate analysis of weight loss at presentation and age at presentation with serum sodium as the dependent variable, serum sodium at presentation was significantly associated with age at presentation $(r=0.69, p<0.005)$ and weight loss at presentation $(r=0.63$., $\mathrm{p}<0.0001$ ) (see figure 2). Urinary electrolytes were collected in only 10 cases with urine osmolarities ranging from 357 to

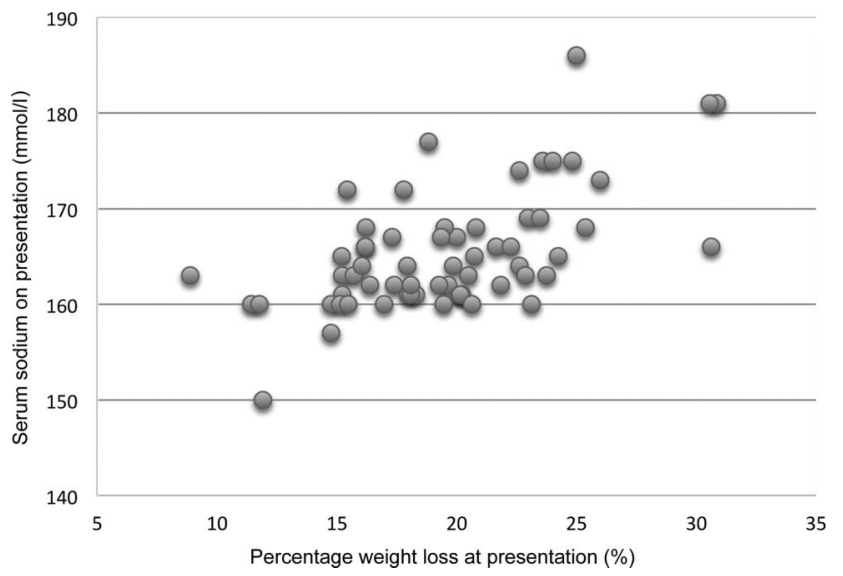

Figure 2 Association of weight loss at presentation or readmission with serum sodium at readmission or presentation in 62 cases of severe neonatal hypernatraemia in the UK and Republic of Ireland, May 2009 to June 2010. In eight cases hypernatraemia worsened in hospital. Two cases had initial sodium values at presentation $<160 \mathrm{mmol} / \mathrm{l}$, but later had values meeting the case definition. 
Table 1 Presenting concerns in 62 cases of severe neonatal hypernatraemia in the UK and Republic of Ireland, May 2009 to June 2010

\begin{tabular}{ll}
\hline Concerns noted at presentation & Number of infants affected \\
\hline Weight loss & 57 \\
Jaundice & 12 \\
Neurological & 6 \\
Lack of stooling & 11 \\
Decreased urine output & 11 \\
\hline
\end{tabular}

$845 \mathrm{mosmol} / \mathrm{l}$, and urine sodium concentrations 44 to $214 \mathrm{mmol} / \mathrm{l}$

At presentation just 25 babies had stooled in the previous $24 \mathrm{~h}$. Presenting concerns are listed in table 1.

Fourteen infants had neurological signs on examination at presentation (confined to drowsiness or lethargy); their serum sodium values exceeded those of infants who did not manifest those signs (median 166 vs $163, \mathrm{p}=0.04$ ). No seizures were noted at presentation or during treatment. Overall, 19 infants presented on the day concerns were noted, with 10, 8, 3, 3 and 2 babies presenting 1,2, 3, 4 and 6 days respectively after concerns were first noted.

\section{Treatment}

A total of 14 babies received bolus infusions of intravenous fluids on admission, and 31 had ongoing intravenous fluid infusions. Babies known to have been treated with intravenous fluids had a higher maximum serum sodium than the remainder (median 166 vs 162, p<0.01). None were dialysed, treated using a central venous line or were transferred to another hospital for care. One baby was ventilated.

The serum sodium fell by a mean $12.7 \mathrm{mmol} / \mathrm{l}$ per $24 \mathrm{~h}$ (SD 6.5). No baby had coma, seizures or other features suggestive of seizures while in hospital. Only three had developed new neurological concerns after admission, and these appeared minor ('jitteriness' and 'irritability').

The median length of stay was 5 days (range 2-14). Ten babies were discharged home breast feeding. All discharged babies had gained weight (median $440 \mathrm{~g}$ ). No deaths occurred.

The survey of weighing practices obtained a response from $75 \%$ of maternity units. Comparison of weighing practices in those units who did, and did not, have a case of SNH did not suggest an intention to perform earlier routine weighing in those units in which cases of SNH did not occur (see table 2).

Six of 49 cases born in England were cared for in fully accredited BFI hospitals. In England 17.5\% of babies are born in BFI hospitals.

Table 2 Weighing practices in maternity units England, Wales and Scotland in 2010

\begin{tabular}{llll}
\hline $\begin{array}{l}\text { First routine } \\
\text { weight }\end{array}$ & $\begin{array}{l}\text { Units overall } \\
(\%)\end{array}=126$ & $\begin{array}{l}\text { Units with cases } \\
(\%) n=33\end{array}$ & $\begin{array}{l}\text { Units with no } \\
\text { cases (\%) } \mathrm{n}=93\end{array}$ \\
\hline D1-5 & $99(78.6)$ & $25(75.8)$ & $74(79.6)$ \\
$\geq D 6$ & $20(15.9)$ & $5(15.2)$ & $15(16.1)$ \\
$\begin{array}{l}\text { No routine } \\
\text { weights }\end{array}$ & $6(4.8)$ & $3(9.1)$ & $3(3.2)$ \\
$\begin{array}{l}\text { No response to } \\
\text { this question }\end{array}$ & $1(0.8)$ & 0 & $1(1.1)$ \\
\hline
\end{tabular}

\section{DISCUSSION}

We have shown that SNH occurs infrequently, and in a 1 year period was associated with no inhospital deaths, and remarkably little short term morbidity, beyond hospital admission and the cessation of breast feeding.

That 61 of 62 of the mothers of these cases had intended exclusive breast feeding is striking. There was an excess of primiparous mothers, section deliveries and higher maternal age-all known associations with feeding difficulty. ${ }^{16}$ As only one baby appears to have developed hypernatraemia after attempts at formula feeding, it is clear that BDAH is the underlying diagnosis in most of the cases we report.

$\mathrm{BDAH}$ as a 'diagnosis' depends on the presence of hypernatraemia, a history of breastfeeding problems and weight loss. It is thus clearly distinguished from salt poisoning where weight loss is not a feature. ${ }^{18}$ The weight loss, and inhospital gain after treatment, in our cases is striking and illustrates missed opportunities for prevention or early intervention.

Other clinical signs and concerns at presentation were noted much less frequently by reporting clinicians. Jaundice was not predominant, and neither stool frequency nor urine output seems to have driven hospitalisation, though the fact that just 25 of the 62 babies had stooled in the previous $24 \mathrm{~h}$ provided an obvious clinical clue that all was not well.

We chose to survey all neonatal hypernatraemia, rather than asking paediatricians to report cases which they attributed to difficulties with feeding. Therefore, we have not missed an opportunity to describe formula feeding associated severe hypernatraemia cases, and this is a significant strength of this study. Such cases appear to be rare. Our study is strengthened by both the BPSU methodology with its high response rate, and the population based methodology, and as such our report of incidence may be more meaningful than a reading of other reports, the interpretation of which may be affected by publication bias. ${ }^{1} \quad 5$ 9-11 13 We used an unambiguous case definition and were able to gather data about most cases. However, our reported incidence might be regarded as a minimal incidence if the 10 non-responding clinicians had real cases they did not, or were not able to, report. Furthermore, although the response rate to the BPSU is high, it is not $100 \%$ and cases missed this way might underestimate the incidence marginally.

We were not able, for reasons of practicality within the BPSU methodology, to collect data on babies presenting with milder hypernatraemia, and are not able to speak with confidence about these cases. It is likely that there are significantly higher numbers of them, but unlikely that their clinical course is more malignant. It is also likely that their numbers are influenced by the approach to diagnostic testing adopted by clinicians.

We were not able to collect data as to the long term outcome of collected cases, though as so few had neurological problems, this might not have been of great concern.

The best study with which to compare our work is that performed by the Dutch Paediatric Surveillance Unit. ${ }^{8}$ They sought hospitalised cases of 'dehydration' as evidenced by weight loss exceeding $10 \%$ of birth weight, and only found six cases ( 1.5/ 100000 live births) where the serum sodium exceeded $150 \mathrm{mmol} / \mathrm{l}$ - a threshold far lower than ours. Many babies with weight loss had no blood tests. This comparison suggests that while the consequences of severe hypernatraemia do not seem severe in the UK, there is more pathology relating to the establishment of feeding here than in some other countries.

It is tempting to speculate that the relatively low rates of initiation and particularly continuation of breast feeding in the UK 
may form part of the explanation for this; where breast feeding is commonplace, it is likely that lay and professional skills in its assessment, and remediation of any difficulties, may be in better supply. There was certainly no excess of cases in accredited BFI hospitals. This is consistent with a hypothesis that BFI accreditation may protect against the development of such severe feeding difficulties. Such a hypothesis deserves further research, but supportive evidence already exists. ${ }^{15}$

It is reassuring that there was so little serious overt associated pathology. Review of reported cases suggests that seizures, possibly during rehydration, might be commonplace. ${ }^{1}{ }^{2}$ We had intended to investigate the maximum safe rate of fall of serum sodium, but this was happily impossible due to the infrequent and minor evidence of neurological dysfunction.

Uncertainty surrounds the maximum desirable rate of fall of serum sodium after slow onset hypernatraemia. ${ }^{18} 19$ Although many of our cases exceed recommended rates of fall (typically quoted at $12 \mathrm{mmol} / \mathrm{l}$ per $24 \mathrm{~h}$ ), no complications of rehydration were observed in this study. As no known complications of treatment occurred among the 40 (lowest $75 \%$ of rates of fall) cases with a maximal rate of fall of serum sodium of less than $17 \mathrm{mmol} / \mathrm{l} / 24 \mathrm{~h}$, it is possible to estimate with $95 \%$ confidence that the rate of seizures among a wider group of babies similarly rehydrated and treated does not exceed $8 \%{ }^{20}$ This may be useful to clinicians managing future cases.

That so little associated serious pathology was seen may of course reflect considerable care and good judgement in management of the rehydration and refeeding. The increasing tendency of paediatricians to use intravenous fluids at higher sodium concentrations would support this speculation.

It is also possible that a proactive approach to weighing and responding to abnormal weights forms part of the modern NHS' preventative response to BDAH-our weighing survey is some, albeit weak, evidence that this may be occurring. Routine weighing is recommended practice by breast feeding and paediatric organisations alike. ${ }^{21} 22$ Our study suggests that policies may not always be adhered to or appropriate actions made in response to abnormal weight loss. This is particularly striking where hypernatraemia worsened in hospital. However, while the cases reported may not represent optimal practice, it is possible that current weighing practice, which in turn may be a consequence of the widespread medical discussion of this issue, led to fewer cases than would otherwise have presented. ${ }^{1245}$

Prevention of this condition, or at least its early detection, should be the priority for any humane healthcare system, both to minimise avoidable harms and distress of hospitalisation, but also to alleviate maternal and infant distress when breast feeding is not establishing well and address remediable concerns, for example, with positioning and attachment. Weighing babies and skilled history taking using recognised tools, including focus on stool output, should be universally practised in the early days, with skilled review of cases until feeding difficulties have been ameliorated, weight gain established and the baby's well-being assured. ${ }^{21-23}$ Such preventative ambition should not be limited to addressing $\mathrm{SNH}$ - the vast numbers of women who cease breast feeding their babies shortly after delivery in the UK will also benefit from the same skillsets, whether their babies have moderate weight loss or not. Readmission of infants to hospital is intrinsically disadvantageous; however, the serious harms reported in association with $\mathrm{SNH}$ appear to be uncommon in the UK.

Acknowledgements We acknowledge the BPSU, supported by the Department of Health, for facilitating the data collection and the reporting clinicians, particularly those who completed the questionnaires. Any views expressed are those of the investigator and not necessarily those of the BPSU or DH. We thank Richard Lynn of the BPSU and Christine Peel who assisted us greatly in running the project. Bradford Teaching Hospitals supported the project by paying the BPSU fees.

Contributors SJO designed the study, ran the data collection and did the primary analysis and wrote the first draft of the paper. KD contributed to the project design and assisted with data collection. $\mathrm{VCr}$ assisted with data collection and conducted the weighing survey and analysed these data. JW contributed to the study design and provided advice about infant feeding data collection. AS carried out the regression analyses and advised on statistical matters. All authors contributed to the final version of the paper. SJO is the guarantor.

Funding This paper presents independent research funded by the National Institute for Health Research (NIHR) under its Collaborations for Leadership in Applied Health Research and Care (CLAHRC) programme. The views expressed in this paper are those of the authors and not necessarily those of the NHS, the NIHR or the Department of Health.

\section{Competing interests None.}

Ethics approval Bradford Research Ethics Committee.

Provenance and peer review Not commissioned; externally peer reviewed.

\section{REFERENCES}

1 Shroff R, Hignett R, van't Hoff W. Life-threatening hypernatraemic dehydration in breastfed babies. Arch Dis Child 2006;91:1025-6.

2 Oddie S, Richmond S, Coulthard M. Hypernatraemic dehydration and breast feeding: a population study. Arch Dis Child 2001;85:318-20.

3 Tjora E, Karlsen LC, Moster D, et al. Early severe weight loss in newborns after discharge from regular nurseries. Acta Pediatr 2010;99;654-7.

4 Macdonald PD, Grant L, Ross SRM. Hypernatraemia in the first few days: a tragic case. Arch Dis Child Fetal Neonatal Ed 2003;88:F350.

5 Laing IA, Wong CM. Hypernatraemia in the first few days: is the incidence rising? Arch Dis Child Fetal Neonatal Ed 2002;87:F158-62.

6 Kaplan JA, Siegler RW, Schmunk GA. Fatal hypernatremic dehydration in exclusively breast-fed newborn infants due to maternal lactation failure. Am J Forensic Med Pathol 1998;19:19-22.

7 Van Dommelen P, van Wouwe JP, Breuning Boers JM, et al. Reference chart for relative weight change to detect hypernatraemic dehydration. Arch Dis Child 2007:92:490-4

8 Pelleboer RA, Bontemps STH, Verkerk PH, et al. A nationwide study on hospital admissions due to dehydration in exclusively breastfed infants in the Netherlands: its incidence, clinical characteristics, treatment and outcome. Acta Paediatr 2009:98:807-11.

9 Iyer NP, Srinivasan R, Evans R, et al. Impact of an early weighing policy on neonatal hypernatraemic dehydration and breast feeding. Arch Dis Child 2008;93:297-9.

10 Cooper WO, Atherton HA, Kahana M, et al. Increased incidence of severe breastfeeding malnutrition and hypernatraemia in a metropolitan area. Pediatrics 1995:96:957-60.

11 Moritz M, Manole MD, Bogen DL, et al. Breastfeeding-associated hypernatremia: are we missing the diagnosis? Pediatrics 2005;116:343-7.

12 Manganaro R, Mani C, Marrone T, et al. Incidence of dehyrdration and hypernatraemia in exclusively breast-fed infants. J Pediatr 2001;139:673-5.

13 Harding D, Moxham J, Cairns P. Weighing alone will not prevent hypernatraemic dehydration. Arch Dis Child Fetal Neonatal Ed 2003:88:F349.

14 Leven LVA, Macdonald PD. Reducing the incidence of neonatal hypernatraemic dehydration. Arch Dis Child 2008:93:9 811.

15 Mikiel-Kostra K, Mazur J. Hospital Policies and their influence on newborn body weight. Acta Paediatr 1999:88:72-5.

16 Regnault N, Botton J, Blanc L, et al. Determinants of neonatal weight loss in term-infants: specific association with pre-pregnancy maternal body mass index and infant feeding mode. Arch Dis Child Fetal Neonatal Ed 2011;96:F217-22.

17 Knowles RL, Friend $H$, Lynn $R$, et al. Surveillance of rare diseases: a public health evaluation of the British Paediatric Surveillance Unit. J Public Health (Oxf) 2012;34:279-86.

18 Haycock GB. Hypernatraemia Diagnosis and management. Arch Dis Child Educ Pract Ed 2006;91:ep8-13.

19 Molteni KH. Initial management of hypernatremic dehydration in the breastfed infant. Clin Pediatrics 1994;33:731-40.

20 Hanley JA, Lippman Hand $A$. If nothing ever goes wrong, is everything all right-interpreting zero numerators. JAMA 1983;243:1743-5.

21 http://www.unicef.org.uk/Documents/Baby_Friendly/Guidance/ Breastfeeding_assessment_tool_comm.pdf?epslanguage=en (accessed 5 March 2013).

22 American Academy of Paediatrics. Breastfeeding and the use of human milk. Pediatrics 2012;129:e827-41

23 Nommsen-Rivers LA, Heinig MJ, Cohen RJ, et al. Newborn wet and soiled diaper counts and timing of onset of lactation as indicators of breastfeeding inadequacy. J Hum Lact 2008;24:27. 